\title{
人胰腺干细胞建系及移植诱导胰岛治疗大鼠 糖尿病
}

效梅 ${ }^{(1)}$, 安立龙 ${ }^{(1)}$, 杨学义 ${ }^{(2)}$, 葛昕 ${ }^{(2)}$, 乔海 ${ }^{(2)}$, 赵婷 ${ }^{2}$ ，马霄飞 ${ }^{(2)}$, 㚞敬庄 ${ }^{(2)}$, 朱梦漾 ${ }^{3}$, 窦忠英 ${ }^{(2)}$
(1) 广东海洋大学农学院动物医学系, 湛江 524088;
(2) 西北农林科技大学，国家干细胞工程技术中心陕西分中心，杨凌 712100;
(3) Department of Pharmacology, Quillen College of Medicine, East Tennessee State University, Johnson City 37614, USA
* 联系人, E-mail: douzhongying@china.com

收稿日期: 2008-03-17; 接受日期: 2008-04-17

国家重点基础研究发展计划(批准号: G1999054301)、国家高技术研究发展计划(批准号: 2002AA216161)、国家自然科学基金(批准号: 39970363)、教育部重点项目(批准号: 103160)、陕西省重点攻关项目(批准号: 2002K01-G3)、广东省自然科学基金(批准号: 04011471) 和广东省教育厅科学基金(批准号: 2003-1009)项目资助

摘要供体不足已成为移植胰岛治疗 I 型和部分 II 型糖尿病的主要障碍, 分离克隆 胰腺干细胞作为种子细胞并诱导其分化为功能性胰岛可提供丰富的移植资源. 本研究 从人流产胎儿胰腺组织分离获得 1 例单克隆胰腺干细胞系. 无菌取流产胎儿胰腺组织, $0.1 \%$ IV 型胶原酶消化分离为单个细胞和细胞团。低糖DMEM $+10 \% \mathrm{FBS}$ 培养, 单个 细胞和细胞团贴壁，原代上皮样胰腺干细胞克隆性生长. $0.25 \%$ 胰蛋白酶 $+0.04 \%$ 已 二胺四乙酸(EDTA)消化传代, 成纤维样细胞和其他细胞逐渐被消除, 上皮样胰腺干细 胞逐渐被纯化. 克隆环篮选, 获得单克隆人胰腺干细胞. 在培养液中添加 $10 \mathrm{ng} / \mathrm{mL}$ 表 皮生长因子(EGF), 单克隆人胰腺干细胞快速生长至单层, 呈铺路石样. 继续传代培 养, 1 例来源于 4 月龄男性流产胎儿胰腺干细胞已传 50 代. 液氮冷冻保存细胞 $1 \times 10^{9}$ 个 以上. 染色体核型分析, 该千细胞系为正常的二倍体细胞. 免疫组织化学反应, 共表 达pdx1, glucagon, nestin及CK19 蛋白，不表达insulin, CD34, CD44 及CD45. RT-PCR检 测, 转录pdx1, glucagon, nestin及 CK19 的mRNA, 不转录insulin. $\beta$-颈基乙醇诱导, 分化 为神经细胞, 免疫组织化学反应表达NF蛋白. 烟酰胺诱导, 分化为DTZ染色阳性, 转 录表达insulin, 分泌insulin和 C肽的功能性类胰岛. 将单克隆人胰腺干细胞体外诱导胰 岛移植在 S T Z 制备的糖尿病大鼠肾囊内, 能降低糖尿病大鼠血糖 水平, 延长寿命.

\section{关键词}

胰腺干细胞

单克隆

分化

移植

人 糖尿病(diabetes mellitus, DM)是危害人类健康的 重大疾病之一，而传统的药物及胰岛素替代疗法却 不能从根本上医治该病。近年来，随着移植技术的不 断成熟和免疫抑制剂的改进，移植胰岛已成为治愈
| 型和部分 || 型糖尿病的有效途径 ${ }^{[1 \sim 4]}$. 但是, 由于 每个临床胰岛移植受体通常需要 2 4 个新鲜尸体胰 腺，胰岛供体短缺的矛盾亦更为突出，制约了利用胰 岛移植治疗糖尿病技术的应用。 
胰腺干细胞(Pancreatic stem cells)是一类存在于 胎儿和成年胰腺组织中的成体干细胞, 能自我更新, 具有多分化潜能，其特点是在自然分化过程中首先 分化为胰腺组织的各种细胞. 采用干细胞组织工程 技术，体外分离克隆胰腺干细胞作为种子细胞，并诱 导其分化为功能性胰岛移植治疗糖尿病, 是解决胰 岛供体短缺的有效途径，意义重大. 目前，分离克隆 胰腺干细胞并将其诱导分化为功能性胰岛移植治疗 糖尿病的研究已成为干细胞组织工程研究的热点之 一 $[5 \sim 10]$. 但是, 由于胰腺干细胞的研究时间较短, 胰 腺干细胞的生物学特性还没有完全定性, 胰腺干细 胞的分离、纯化及扩增方法均处于探索中, 获得的胰 腺干细胞的鉴定结果也不尽一致，世界范围内还没 有建立公认的人胰腺干细胞系 $[\underline{11} \underline{13}]$.

本研究从人流产胎儿胰腺组织中分离获得 1 例 单克隆胰腺干细胞系, 通过染色体核型分析、免疫组 织化学反应、RT-PCR 及体外诱导分化实验对其生物 学特性进行了分析, 将其体外诱导胰岛移植在链脲 菌素(streptozocin, STZ)制备的糖尿病模型大鼠肾囊 内, 初步探讨了治疗大鼠糖尿病的效果. 该研究为进 一步开展胰腺干细胞及其诱导胰岛移植治疗糖尿病 提供了新方法和种子细胞.

\section{1 材料与方法}

\section{1 胰腺干细胞的分离与单克隆}

研究材料为 4 6 月龄水囊引产的人胎儿胰腺组 织(来源于西安某医院, 并征得家属同意). 无菌取新 鲜胎儿胰腺组织, PBS(-)清洗后, 将组织剪碎至 1 $\mathrm{mm}^{3}, 0.1 \%$ IV 型胶原酶(Sigma)消化 20 40 min, 收集 单个细胞和细胞团, 低糖DMEM $+3.7 \mathrm{~g} / \mathrm{L} \mathrm{NaHCO}_{3}+$ $10 \% \mathrm{FBS}+0.08 \mathrm{~g} / \mathrm{L}$ 青霉素 $+0.1 \mathrm{~g} / \mathrm{L}$ 链霉素(低糖 DMEM, $\mathrm{NaHCO}_{3}$ 及FBS, Sigma)培养液培养. 当原代 上皮样胰腺干细胞长出后, $0.25 \%$ 胰蛋白酶 $+0.04 \%$ EDTA(胰蛋白酶、EDTA, Sigma)消化，传代. 在传代 中逐渐消除成纤维样细胞和其他细胞，纯化上皮样胰 腺干细胞. 采用克隆环篮选出典型的单克隆人胰腺干 细胞, 培养液中添加 $10 \mathrm{ng} / \mathrm{mL}$ EGF(Sigma)扩增培养. 活力较好的单克隆人胰腺干细胞液氮冷冻保存.

\section{2 染色体核形分析}

第 $10,20,30,40$ 及 50 代的单克隆人胰腺干细胞
扩增至单层, $0.2 \mu \mathrm{g} / \mathrm{mL}$ 秋水仙素(Sigma)处理 $5 \mathrm{~h}$, $0.075 \mathrm{~mol} / \mathrm{L} \mathrm{KCl}$ 低渗 $25 \mathrm{~min}$, 甲醇-冰醋酸固定, 制 备染色体标本，进行核型分析.

\section{3 细胞免疫组织化学反应}

为了确定单克隆人胰腺干细胞的表达特征，采 用 SP 法分别进行了 pdx1, glucagon, insulin, CK19, nestin, CD 34, CD44 及 CD45 免疫组织化学反应. 单 克隆人胰腺干细胞扩增至单层, $4 \%$ 多聚甲醛固定 10 $\min$, 山羊血清室温作用 $15 \mathrm{~min}$, 封闭. 加一抗, $4^{\circ} \mathrm{C}$ 过夜. 一抗分别为 $1: 500$ 稀释的小鼠抗家兔胰肠同 源域因子 1(pancreatic and duodenal homeobox factor 1, pdx1)抗体、1：2000 稀释的小鼠抗小鼠 glucagon 抗 体、1：200 稀释的豚鼠抗牛 insulin 抗体、1:50 稀 释的小鼠抗人 CK19 抗体, 均购自 Sigma; $1: 100$ 稀 释的家兔抗小鼠 nestin 抗体、小鼠抗人 CD34、CD44、 CD45 工作液, 均购自 Bosd. 洗涤, 加山羊抗小鼠生 物素化 Ig G(SP-9000)作为二抗, $37^{\circ} \mathrm{C}$, 反应 $15 \mathrm{~min}$. 洗涤, 加辣根酶标记链霉卵白素工作液 (S-A/HRP), $37^{\circ} \mathrm{C}$, 反应 $15 \mathrm{~min}$. 洗涤, 加显色剂 DAB(ZLI-9033) 显色. 反应阳性细胞为深红色. SP 试剂盒, DAB 显色 试剂盒, 购自北京中山生物科技有限公司. 小鼠胎儿 成纤维细胞作阴性对照.

\subsection{RT-PCR}

单克隆人胰腺干细胞生长至单层, Trizol 分离液 (Gibco)提取细胞总 RNA，合成 cDNA 后，PCR．根据 GenBank 中人 pdx1, insulin, glucagon, nestin, CK19 及 $\beta$-actin 序列设计引物, 北京 AuGCT 生物公司合成. 胰肠同源域因子 1(pdx1, $441 \mathrm{bp})$ 引物: 正链引物 L 5'-TGGCGCACCTTCACCACCAC-3' ; 反链引物 H 5' -CCTGCTCAGGCTCCGCGACC-3'。胰岛素(insulin, 212 bp)引物: 正链引物 L 5' -CAGCCGCAGCCTTTGTGAAC-3'; 反链引物 H 5' - AATGCCACGCTTCTGCAGGG-3'. 胰高血糖素(glucagon, 353 bp)引物: 正链引物 L 5' -AGCATTTACTTTGTGGCTGG-3'; 反链引物 H 5' ATGAATTCCTTGGCAGCTTG-3'．巢蛋白(nestin, 334 bp)引物: 正链引物 L 5'-TTCTGTGAGTGTCAGTGTCC-3'; 反链引物 H 5' -CTCAGAGACTAGCGGCATT- 
C-3'. 角蛋白 19(CK19, 490 bp)引物：正链引物 L 5'-GAGTACCAGCGGCTCATG-3'; 反链引物 H 5' -GCTGCGGTAGGTGGCAAT- $3^{\prime}$. 内源对照 $\beta$-肌动蛋白 $(\beta$ actin, 268 bp)引物: 正链引物 L 5'-CTCCATCCTGGC CTCGCTGT-3'; 反链引物 H 5'-GCTGTCACCTTCACCGTTCC-3'.

PCR 反应条件为 $94^{\circ} \mathrm{C}$ 变性 $3 \mathrm{~min}, 93^{\circ} \mathrm{C}$ 变性 $1 \mathrm{~min}$, $72^{\circ} \mathrm{C}$ 延伸 $1 \mathrm{~min}$, 循环 35 次, $72^{\circ} \mathrm{C}$ 再延伸 $10 \mathrm{~min} .1 .0 \%$ 琼脂糖凝胶电泳。分离的原代人胎儿胰岛细胞作 $\mathrm{pdx} 1$, insulin 及 glucagon 阳性对照，原代人胎儿脑神经 细胞作 nestin 阳性对照, 原代人胎儿皮肤上皮细胞作 CK19 阳性对照. 小鼠胎儿成纤维细胞作阴性对照.

\section{5 向神经细胞诱导分化}

单克隆人胰腺干细胞扩增至单层, 加含 1 $\mathrm{mmol} / \mathrm{L} \beta$-颈基乙醇(Sigma)的低糖 DMEM 诱导液诱 导培养 $24 \mathrm{~h}$, 换加神经细胞条件培养液(由本实验室 制备. 培养人流产胎儿大脑神经细胞, 收集培养液, 离心 $800 \times g, 30 \mathrm{~min} .0 .22 \mu \mathrm{m}$ 滤膜过滤, $-20^{\circ} \mathrm{C}$ 保存, 备用). 继续诱导培养 $48 \mathrm{~h}$. 家兔抗家兔神经丝蛋白 (nerve filament, NF, Bosd)抗体免疫组织化学反应. 方 法同 1.3 小节. 单克隆人胰腺干细胞作阴性对照.

\section{6 向胰岛诱导分化}

单克隆人胰腺干细胞接种在铺有 $0.1 \%$ 明胶 (Sigma)的培养血内, 扩增培养 3 天, 加含 $10 \mathrm{mmol} / \mathrm{L}$ 烟酰胺(Sigma)的高糖 DMEM(Sigma)诱导液诱导培养 25 天. 分别采用双硫腙染色法、RT-PCR 及放射免疫 实验对诱导结果进行检测.

(1) 双硫腙染色. 方法参照文献[14]. 当干细胞 诱导培养 10,15 及 25 天时, 分别进行双硫腙 (dithizone, DTZ, Sigma)染色. 若干细胞已分化为胰岛 $\beta$ 细胞, 则细胞内含大量 $\mathrm{Zn}^{+}, \mathrm{DTZ}$ 特异性与 $\mathrm{Zn}^{+}$结合, 形成深红色络合物. DTZ毒性小, 可进行活细胞染色, 去掉染色液, $6 \mathrm{~h}$ 后, 红色消失, 细胞仍能正常生长. 分离的原代人胎儿胰岛细胞作阳性对照, 单克隆人 胰腺干细胞作阴性对照.

(2) RT-PCR. 当干细胞诱导培养 25 天时, Trizol 分离液提取诱导胰岛细胞总 RNA, 合成 cDNA 后,
PCR. 根据 GenBank 中人 insulin 和 $\beta$-actin 序列设计 引物, 北京 AuGCT 生物公司合成. 胰岛素和内源对 照 $\beta$ 肌动蛋白的引物设计及 PCR 同 1.4 小节. 分离的 原代人胎儿胰岛细胞作阳性对照.

(3) 肤岛素和 $\mathrm{C}$ 肽放射免疫实验. 将单克隆人胰 腺干细胞接种在 3 个 6 孔板内, 细胞接种数为 $5.0 \times 10^{5}$ 个 孔, 扩增培养 3 天, 换用诱导液诱导培养 25 天. 弃诱 导液, 无糖DMEM洗 3 遍, 1 板每孔加 $1 \mathrm{~mL}$ 无糖DMEM, 第 2 板每孔加 $1 \mathrm{~mL}$ 含 $5.50 \mathrm{mmol} / \mathrm{L}$ 葡萄糖(Sigma)的 DMEM刺激液, 第 3 板每孔加 $1 \mathrm{~mL}$ 含 $25.00 \mathrm{mmol} / \mathrm{L}$ 葡萄糖的DMEM刺激液, $37^{\circ} \mathrm{C}$, 培养 $2 \mathrm{~h}$, 分别收集 各孔培养液. 所有收集液离心 $(800 \times \mathrm{g}, 10 \mathrm{~min}),-20^{\circ} \mathrm{C}$ 保 存. 采用放射免疫法测定诱导胰岛insulin和C肽的释放 量(人insulin、C肽放射免疫分析药盒，北京北方生物技 术研究所). 测定结果进行 $t$ 检验.

\section{7 体外诱导胰岛移植}

体重约 $(250 \pm 20) \mathrm{g}$ 的 Sprague-Dawley 正常雄性 成年大鼠 30 只(购自第四军医大学实验动物中心). 随 机取 6 只大鼠, 腹腔注射剂量为 $55 \mathrm{mg} / \mathrm{kg}$ 体重的 0.1 $\mathrm{mol} / \mathrm{L}$ 柠檬酸-柠檬酸钠缓冲液 $(\mathrm{pH}$ 4.4) 作阴性对照. 其余 24 只大鼠腹腔注射相同剂量的 $2 \%$ 链脲菌素 (streptozocin, STZ, Sigma, $0.1 \mathrm{~mol} / \mathrm{L}$ 柠檬酸-柠檬酸钠 缓冲液( $\mathrm{pH} 4.4$ )稀释), 注射后 $48 \mathrm{~h}, 5$ 天及 8 天, 断尾 采血, 用稳步倍加型血糖仪(LIFESCAN Co.)测定血 糖. 随机取 3 次血糖均高于 $16.65 \mathrm{mmol} / \mathrm{L}$ 的大鼠, 6 只用作糖尿病阳性对照, 12 只用于胰腺干细胞体外诱 导胰岛移植抗大鼠糖尿病实验.

单克隆人肤腺干细胞培养在 6 孔板内, 细胞量约 为 $1.0 \times 10^{4}$ 个妚. 扩增培养 3 天后, 诱导培养 25 天. 方 法同 1.6 小节. DTZ染色检测后，用细胞刮刮下阳性孔 细胞, 高糖DMEM稀释每孔细胞至 $100 \mu \mathrm{L}$ 用于移植.

12 只用作诱导胰岛细胞移植的糖尿病大鼠腹腔 注射麻醉剂, 待大鼠进入麻醉状态后, 左侧肋骨下缘 做 $1.5 \mathrm{~cm}$ 左右的横切口，暴露肾脏，向肾囊内缓慢注 入剂量为 $100 \mu \mathrm{L} /$ 只的诱导胰岛细胞悬液. 注射完毕, 肾脏复位，缝合. 6 只糖尿病阳性对照大鼠按同样的 方法在肾囊内注射剂量为 $100 \mu \mathrm{L} /$ 只的高糖 DMEM.

实验大鼠(6 只正常对照大鼠、6 只糖尿病阳性对照 大鼠及 12 只诱导胰岛移植大鼠)均每 10 天断尾测血糖 
1 次, 记录大鼠的血糖浓度和死亡率，以评价单克隆人 胰腺干细胞体外诱导肤岛移植抗大鼠糖尿病的效果.

\section{2 结果与分析}

\section{1 单克隆人胰腺干细胞系的建立}

采用上述分离扩增方法, 本研究建立 1 例单克隆 人胰腺干细胞系. IV 型胶原酶消化分离人胎儿胰腺组 织, 获得单个细胞和细胞团. 低糖DMEM有血清培养, 单个细胞和细胞团 $24 \mathrm{~h}$ 内贴壁, 3 5 天后，原代上皮 样肤腺干细胞开始克隆性生长(图 1(a)). 胰蛋白酶消 化传代, 成纤维样细胞和其他细胞在传代中逐渐被消 除，上皮样胰腺干细胞逐渐被纯化(图 1(b)). 采用克隆 环篮选, 从已纯化的胰腺干细胞中获得典型的单克隆 细胞. 培养液中添加EGF, 单克隆胰腺干细胞迅速扩 增, 呈铺路石样(图 1(c)). 传代培养, 胰腺干细胞数量 不断增加. 1 例来源于 4 月龄男性胎儿的单克隆胰腺干 细胞已传 50 代, 液氮冷冻保存干细胞 $1 \times 10^{9}$ 个以上.

\section{2 单克隆人胰腺干细胞染色体核型分析}

第 $10,20,30,40$ 及 50 代的单克隆人胰腺干细胞
核型分析显示，染色体均为正常的二倍体 $(2 n=46)$. 其中, 第 50 代的单克隆人胰腺干细胞染色体核型为 中部着丝粒染色体 5 对, 亚中部着丝粒染色体 7 对, 端部着丝粒染色体 6 对, 小染色体 4 对, 性染色体 1 对(图 2(a)和(b)).

\section{3 单克隆人胰腺干细胞的表达特征}

免疫组织化学反应显示单克隆人胰腺干细胞共 表达 pdx1, glucagon, nestin 及 CK19 蛋白(图 3(a) (d)), 且 glucagon 仅在细胞核内表达, 不表达 insulin, CD34, CD44 及 CD45.

RT-PCR(图 4)进一步证实该干细胞转录表达 pdx1, glucagon, nestin 及 CK19 的 mRNA, 不转录表达 insulin.

\section{4 单克隆人胰腺干细胞的分化特性}

体外定向诱导, 单克隆人胰腺干细胞分化为神 经细胞和功能性类胰岛.

（1）分化为神经细胞. $\beta$-颈基乙醇诱导 $24 \mathrm{~h}$, 细 胞收缩，并形成突起，呈双极或多极胞体(图 5(a)). 换 用神经细胞条件培养液继续诱导 $48 \mathrm{~h}$, 神经样纤维进
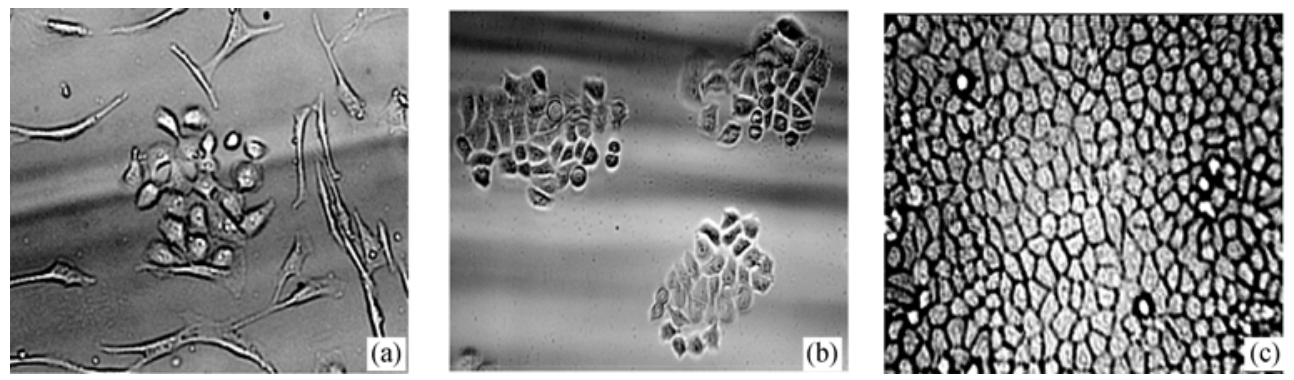

图 1 原代人胰腺干细胞单克隆生长 $((\mathbf{a}), \times 100)$; 纯化的人胰腺干细胞 $((\mathbf{b}), \times 100)$; 单克隆人胰腺干细胞生长至单层时呈铺 路石样 $((\mathbf{c}), \times 100)$
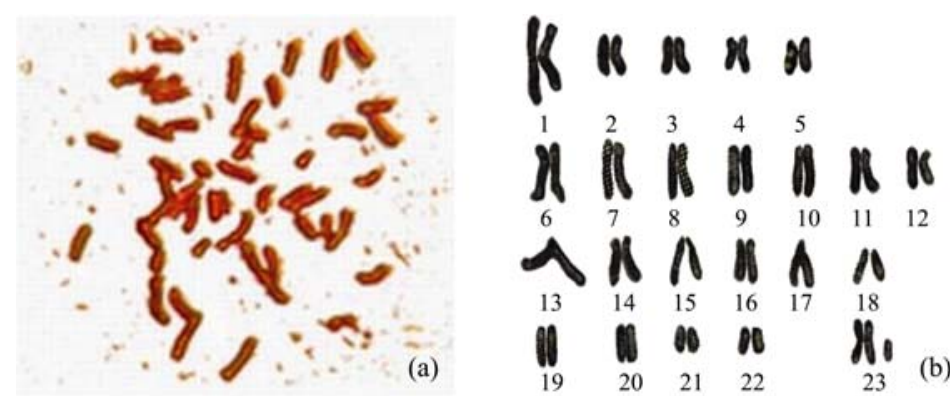

图 2 第 50 代单克隆人胰腺干细胞染色体 $((a), \times 400)$ 与染色体核型(b) 

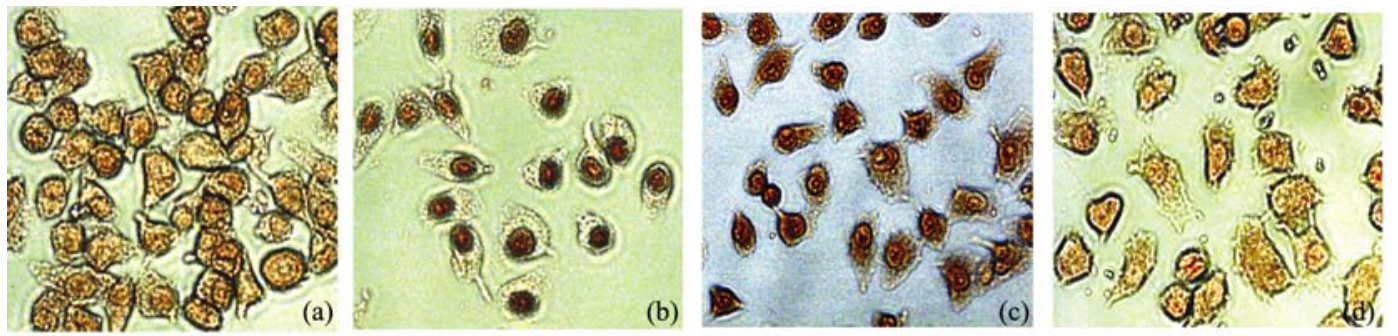

图 3 免疫组织化学反应, 单克隆人胰腺干细胞共表达胰肠同源域 $1((a), \times 200) 、$ 胰高血糖素 $((b), \times 200) 、$ 巢蛋白 $((c), \times 200)$ 及角蛋白 19((d), ×200)

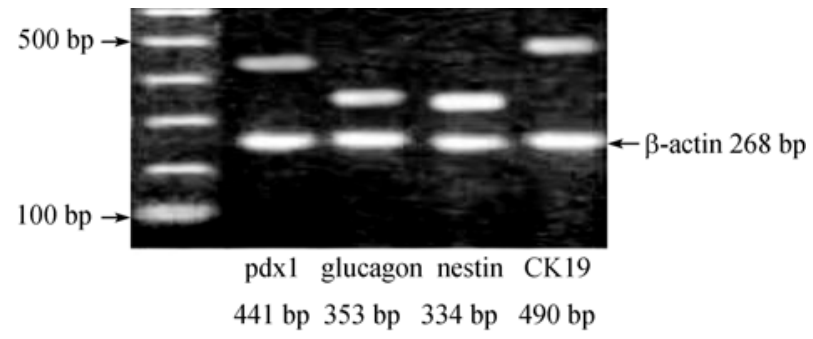

图 4 RT-PCR, 单克隆人胰腺干细胞转录表达胰肠同源域

1、胰高血糖素、巢蛋白及角蛋白 19 的 mRNA

一步生长, 连结成网状(图 5(b)). 免疫组织化学反应 表达 NF 蛋白(图 5(c)).
（2）分化为功能性胰岛. 在向胰岛分化的过程中, 干细胞逐渐由上皮样变为圆形，并聚集成类胰岛细 胞团. 诱导 15 天时, 个别类胰岛细胞团 DTZ 染色呈 深红色(图 5(d)). 诱导 25 天时，大部分类胰岛细胞团 DTZ 染色呈深红色(图 5(e)). 去染色液, 加原诱导液 培养 $6 \mathrm{~h}$, 着色胰岛细胞团红色全部消失. 图 5(f)是 4 月龄人胎儿胰腺组织消化后, 原代胇岛 DTZ 染色阳 性对照.

RT-PCR 进一步证实诱导培养 25 天时, 干细胞 分化形成的类胰岛 $\beta$ 细胞转录表达 insulin 的 mRNA (图 6).

放射免疫实验显示 $5.0 \times 10^{5}$ 个干细胞扩增 3 天,
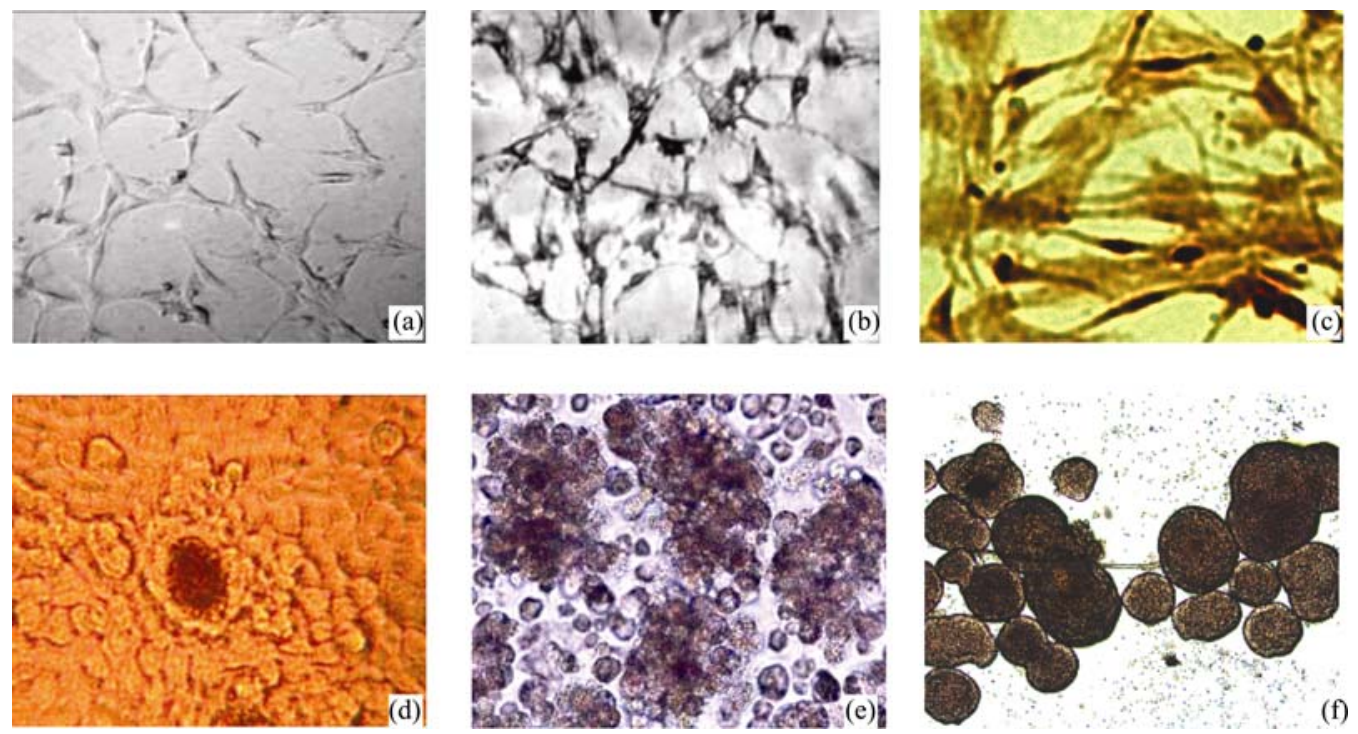

图 5 单克隆人胰腺干细胞向神经细胞诱导 $24 \mathrm{~h}$ 时, 大部分细胞形成双极或多极胞体((a), $\times 100)$; 继续诱导 $48 \mathrm{~h}$ 时, 神经样 纤维进一步生长, 连结成网状((b), ×100), 免疫组织化学反应表达神经丝蛋白((c), × 200)

向胰岛诱导 15 天时, 少数细胞双硫腙染色阳性 $((\mathrm{d}), \times 100)$; 诱导 25 天时, 大部分细胞双硫腙染色阳性 $((\mathrm{e}), \times 100)$; (f)为 4 月龄人胎儿胰 腺组织消化后原代胰岛双硫腙染色阳性对照 $\times 50$ 
诱导培养 25 天, 分别用 $1 \mathrm{~mL}$ 含 $0.00,5.50$ 及 25.00 $\mathrm{mmol} / \mathrm{L}$ 葡萄糖的刺激液培养 $2 \mathrm{~h}$, insulin 释放量依次 是 $0.00,(6.52 \pm 0.37)$ 及 $(13.94 \pm 0.22) \mu \mathrm{IU} / \mathrm{mL}, \mathrm{C}$ 肽 释放量依次是 $0.00,(0.27 \pm 0.08)$ 及 $(0.34 \pm 0.05)$ $\mathrm{pmol} / \mathrm{L}$. 这表明单克隆人胰腺干细胞体外诱导胰岛 释放 insulin 和 $\mathrm{C}$ 肽均依赖于糖刺激, insulin 和 $\mathrm{C}$ 肽释 放量随糖刺激浓度升高显著增加 $(0.01<P<0.05)$, 且 insulin 和 C 肽释放量呈正相关.

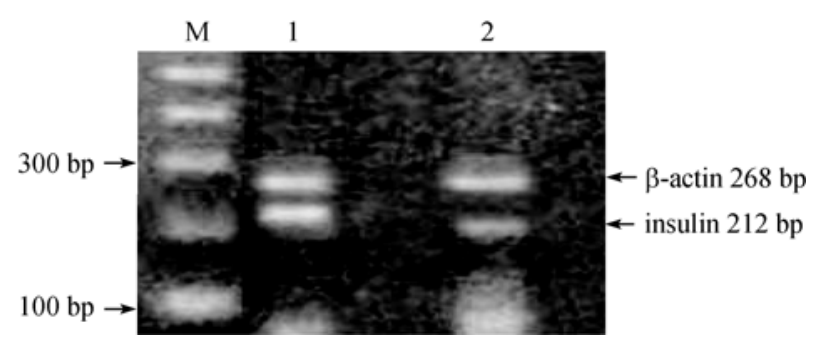

图 6 RT-PCR, 单克隆人胰腺干细胞分化的类胰岛细胞转 录表达 insulin

M: Marker DL 500; 泳道 1: 原代人胎儿胰岛细胞 RT-PCR 阳性对 照结果；泳道 2：诱导胰岛细胞 RT-PCR 结果

\section{5 单克隆人胰腺干细胞体外诱导胰岛移植抗大 鼠糖尿病}

在为期 67 天的移植实验中, 12 只诱导胰岛细胞 移植大鼠，除 2 只移植后 3 天内死亡(感染所致)外, 其余存活了 54 67 天. 存活大鼠血糖水平从 18.93 $25.78 \mathrm{mmol} / \mathrm{L}$ 降至 $6.32 \sim 11.47 \mathrm{mmol} / \mathrm{L} .6$ 只糖尿病对 照大鼠注射 DMEM 诱导液后，1只 3 天内死亡(感染 所致)，5只存活了 16 42 天，血糖水平持续在 18.72 25.96 mmol/L. 6 只正常对照大鼠血糖基本保持 在 $3.54 \sim 5.83 \mathrm{mmol} / \mathrm{L}$, 死亡率为 0 . 证实移植单克隆 人胰腺干细胞体外诱导胰岛细胞能降低糖尿病大鼠 血糖水平，延长其寿命. 图 7(a)和(b), 糖尿病大鼠肾 囊内移植单克隆人胰腺干细胞体外诱导胰岛. 图 7(c) 为实验大鼠(正常对照大鼠、糖尿病对照大鼠及肾囊 内移植诱导胰岛大鼠)血糖浓度的变化.

\section{3 讨论}

体外分离克隆胰腺干细胞，定向诱导胰腺干细 胞分化为功能性胰岛移植治疗糖尿病，意义重大. 虽 然目前世界范围内还没有建立公认的胰腺干细胞系,
但是，已有的研究表明分离克隆人、小鼠及猪等胰腺 干细胞，定向诱导其分化为功能性胰岛，将这些诱导 胰岛移植给糖尿病模型鼠类能抗糖尿病. 1997 年, Peck领导的研究小组 ${ }^{[15]}$ 首次报道采用 $\mathrm{V}$ 型胶原酶不 完全消化非肥胖性糖尿病(Nonobese diabetic，NOD) 小鼠胰腺组织, 手工分离胰导管, 无血清培养, 胇导 管上皮样细胞克隆形成单层. 加入糖尿病供体血清 诱导培养，上皮样细胞分化为胰岛前体细胞，聚集形 成球状结构. 加入葡萄糖继续诱导, 构成球状结构的 细胞进一步分化形成类胰岛细胞团, insulin和 glucagon抗体免疫反应呈弱阳性. 以后，该研究小 组 ${ }^{[5,16]}$ 又相继报道来源于 NOD 小鼠的胰腺干细胞已 传 3 年多, 烟酰胺诱导, 肤导管上皮样细胞分化形成 包含 $\alpha, \beta$ 及 $\delta$ 细胞的功能性胰岛细胞团. 葡萄糖刺激, 诱导胰岛释放insulin. 将诱导胰岛细胞团移植在NOD 成年小鼠肾囊内, 能降低NOD小鼠血糖水平, 抗糖尿 病. Bonner-Weir等人 ${ }^{[6]}$ 采用 $\mathrm{V}$ 型胶原酶消化成年人胰 腺组织, 密度梯度离心, CMRL 1066 有血清扩增培养, 胰导管上皮样细胞形成单层. 采用鼠类基质膜成分 诱导培养 3 4 周，上皮样细胞形成类胰岛细胞团， DTZ染色阳性，且分泌insulin. Zulewski等人 ${ }^{[17]}$ 用V型 胶原酶消化成年大鼠或人的胰腺组织获得胰岛, RPMI1640 培养 $96 \mathrm{~h}$, 手工挑取悬浮胰岛, 继续培养, 一些细胞脱离胰岛, 贴壁生长至单层. 挑出单层中的 5 10个细胞进行亚克隆, 细胞不断扩增. 来源于大鼠 胰岛的细胞 8.5 个月扩增了 10 倍，来源于人胰岛的细 胞 8 个月扩增了 7 倍. 体外诱导培养, 这些亚克隆细 胞分化为胰腺的内、外分泌部细胞. $\mathrm{Wu}$ 等人 ${ }^{[18]}$ 扩增培 养来源于人胎儿胰腺组织表达nestin的细胞至 20 代, 体外定向诱导，分化为胰岛细胞，将诱导胰岛移植给 STZ制备的糖尿病小鼠，能抗小鼠糖尿病.Yatoh等人 [19]采用CA19-9 抗体篎选，获得纯化的成年人胰导管 细胞. 添加基质细胞成分体外定向诱导，这些纯化的 胰导管细胞分化为insulin分泌细胞. 本研究采用与其 他研究不同的分离、克隆方法，建立 1 例单克隆人胰 腺干细胞系，传 50 代. 烟酰胺体外定向诱导，单克隆 人胰腺干细胞分化为DTZ染色阳性，且分泌insulin和 $\mathrm{C}$ 肽的功能性类胰岛. 在建立单克隆人胰腺干细胞系 的过程中, 本研究组认为纯化胰腺干细胞是关键步 骤. 不同的细胞其贴壁性和对消化 

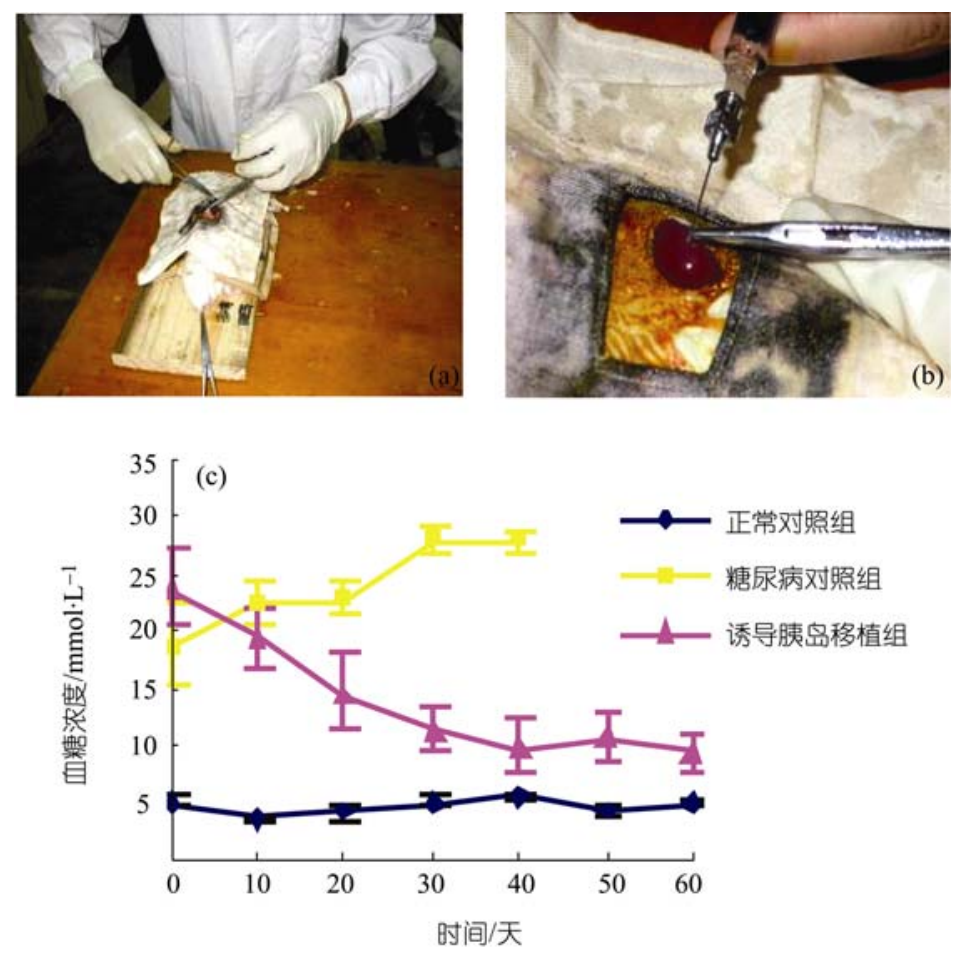

图 7 糖尿病大鼠肾囊内移植单克隆人胰腺干细胞诱导胰岛

(a), (b) 为实验过程; (c) 为实验大鼠(正常对照大鼠、糖尿病对照大鼠、诱导胰岛移植大鼠)血糖浓度的变化

酶的敏感性不同, 可依此逐渐去除成纤维样细胞和 其他细胞, 纯化出呈单克隆生长的胰腺干细胞. 从细 胞形态、扩增及分化为功能性类胰岛的生物学特性看, 本研究分离的单克隆人胰腺干细胞更类似于 Peck 等 人 $[5,15,16]$ 分离获得的NOD小鼠胰腺干细胞.

尽管目前胰腺干细胞的标记物还没有完全确定, 研究者认为在胰腺发育过程中，一些影响胰腺细胞 发育命运的因子如 pdx $1, H N F 3 \beta$ 及 neurogenin 3 等 可作为胰腺干细胞的候选标记物. 除此之外, 胰腺干 细胞的多分化潜能还使其共表达胰腺多种终末细胞 表达物，甚至共表达来源于其他胚层细胞的表达 物 ${ }^{[20,21]}$. 如, Teitelman等人 ${ }^{[22]}$ 报道在小鼠胰腺发育早 期，胰腺干细胞共表达insulin, glucagon及神经原蛋白 tyrosine hydroxylase. Yang等人 ${ }^{[23]}$ 的研究表明在人胰 腺发育早期, 胰导管上皮样细胞共表达insulin, glucagon, somatostatin及 CK19. Bonner-Weir等人苜体外 培养来源于成年人胰导管共表达 CK19 和 $\mathrm{pdx} 1$ 的胰 腺上皮样细胞分化为分泌 insulin 的 $\beta$ 细胞. Maria-Engler等人 ${ }^{[24]}$ 体外长期培养来源于人胰岛表达
神经干细胞标记物 nestin的细胞转分化为共表达 nestin和 insulin的胰岛祖细胞后，进而分化为 $\beta$ 细胞. 本研究为了确定单克隆人胰腺干细胞的表达特征, 参照很多研究结果, 在免疫组织化学反应实验中, 分 别选用pdx1, glucagon, insulin, nestin及CK19 多种胰 腺干细胞候选标记物对其细胞表达特性进行了检测. 同时，还选用造血干细胞标记物CD34 和骨髓间充质 干细胞标记物CD44 和CD45 对其进行排除鉴定. 结 果显示，单克隆人胰腺干细胞共表达pdx1, glucagon, nestin及CK19 蛋白, 不表达insulin, CD34, CD44 及 CD45 蛋白. RT-PCR, 进一步确定其转录pdx1, nestin, glucagon及CK19 的mRNA, 不转录insulin. pdx1 在胰 腺发育早期，整个胰腺细胞表达，发育后期，则限于 胰岛细胞表达，到了成年期，仅 $\beta$ 细胞表达. 而选择 性剔除成年小鼠胰岛细胞 $p d x 1$ 基因，小鼠胰岛遭破 坏，引发糖尿病。因此，常用作胰腺干细胞标记 物 ${ }^{[6,10,19,25]}$. glucagon虽然是胰岛 $\alpha$ 细胞的分泌物, 但 研究表明胰岛祖细胞共表达 glucagon ${ }^{[5,8,15,16,22,23]}$. CK19 是上皮干细胞标记物, nestin 是神经干细胞标 
记物，同时它们也是胰腺干细胞的共表达物 ${ }^{[6,19,24]}$. 由此, 可以确定本研究从人胎儿胰腺组织中分离的 这例单克隆人胰腺干细胞系是与胰岛、上皮及神经组 织分化有关的多潜能干细胞. 值得一提的是, Taguchi 和Otsuki ${ }^{[26]}$ 采用共聚焦激光显微(confocal laser microscopy)技术研究证实, 在成年大鼠胰导管膨大部 和胰岛中均存在共表达 $\mathrm{pdx} 1$ 和nestin的细胞，而这类 细胞存在于胰导管时还共表达CK19, 因此, 他们认 为存在于成年大鼠胰导管中这种共表达 $\mathrm{pdx} 1, \mathrm{CK} 19$ 及nestin的细胞是胰腺干细胞的重要候选细胞，迁移 入胰岛后，该细胞进一步分化为共表达 $\mathrm{pdx} 1$ 和nestin 的胰岛细胞. 这一研究结果为本研究首次获得 1 例共 表达pdx1, glucagon, nestin及CK19 蛋白的单克隆人胰 腺干细胞系提供了佐证.

胰腺干细胞是存在于胰腺组织的成体干细胞, 除能优先分化为胰腺组织的各种细胞外, 还具有分 化为其他胚层来源终末细胞的多分化潜能. Zulewski 等人 ${ }^{[17]}$ 报道在诱导液中添加葡萄糖，能促使来源于 成年大鼠胰岛表达nestin的干细胞分化为 $\beta$ 细胞; 添 加HGF, ctivin-A及 exendin-4, 则促使干细胞分化为 $\alpha, \beta$ 及胰岛其他细胞，甚至肝脏细胞. Danner等人 [27] 采用诱导胚胎干细胞分化为胚体的方法, 悬滴培养 来源于成年大鼠胰腺外分泌部的干细胞分化为类卵 母细胞. Seaberg等人 ${ }^{[28]}$ 体外诱导成年小鼠胰腺干细 胞分化为胰腺细胞和神经细胞. Davani等人 ${ }^{[29]}$ 体外诱 导表达 CD73, CD 90 及 CD105 的人胰岛间充质祖细胞 分化为脂肪细胞、软骨细胞及成骨细胞, 将祖细胞移 植在小鼠体内，自然分化为胰岛内分泌细胞. Kruse等 人 ${ }^{[30]}$ 报道来源于成年大鼠胰腺外分泌部的干细胞体 外诱导能分化为 3 个胚层的细胞, 形成类畸胎瘤. 本 研究结果证实来源于人胎儿胰腺组织的单克隆干细 胞同样具有多分化潜能. 体外诱导, 至少能分化为胰 岛细胞和神经细胞.

综上，本实验室已建立了 1 例共表达 pdx 1 , glucagon, nestin 及 CK19 蛋白的单克隆人胰腺干细胞系. 体外定向诱导, 分化为功能性类胰岛和神经细胞. 将 诱导类胰岛移植给糖尿病模型大鼠，能抗大鼠糖尿 病. 该实验为进一步研究人类和其他哺乳动物胰腺 干细胞及其诱导胰岛移植治疗糖尿病提供了种子细 胞和新方法.

\section{参考文献}

1 Shapiro A M, Lakey J R, Ryan E A. Islet transplantation in seven patients with type 1 diabetes mellitus using a glucocorticoid-free immunosuppressive regimen. N Engl J Med, 2000, 343(4): 230-238[DOI]

2 Robertson R P. Islet transplantation as a treatment for diabetes - a work in progress. N Engl J Med, 2004, 350(7): 694-705[DOI]

3 Shapiro A M, Ricordi C R, Hering B J, et al. International trial of the Edmonton protocol for islet transplantation. N Engl J Med, 2006, 355(13): $1318-1330 \underline{\text { DOI] }}$

4 Lakey J R, Kin T, Warnock G L, et al. Long-term graft function after allogeneic islet transplantation. Cell Transplant, 2007, 16(4): $441-446$

5 Ramiya V K, Maraist M, Arfors K E, et al. Reversal of insulin-dependent diabetes using islets generated in vitro from pancreatic stem cells. Nat Med, 2000, 6(3): 278-282[DOI]

6 Bonner-Weir S, Taneja M, Weir G C, et al. In vitro cultivation of human islets from expanded ductal tissue. Proc Natl Acad Sci USA, 2000, 97(14): 7999-8004[DOI]

7 Suzuki A, Nakauchi H, Taniguchi H. Prospective isolation of multipotent pancreatic progenitors using flow-cytometric cell sorting. Diabetes, 2004, 53(8): 2143-2152[DOI]

8 Xiao M, An L L, Ge X, et al. Isolation, clone and identify of stem cells derived from abortive fetal pancreas. Tissue Eng, 2006, 12(4): 1047

9 Oshima Y, Suzuki A, Kawashimo K, et al. Isolation of mouse pancreatic ductal progenitor cells expressing CD133 and c-Met by flow cytometric cell sorting. Gastroenterology, 2007, 132(2): 720-732[DOI]

10 Nelson S B, Schaffer A E, Sander M. The transcription factors Nkx6.1 and Nkx6.2 possess equivalent activities in promoting beta-cell fate specification in Pdx1+ pancreatic progenitor cells. Development, 2007, 134(13): 2491-2500[DOI]

11 Dor Y, Brown J, Martinez O I, et al. Adult pancreatic $\beta$-cells are formed by self-duplication rather than stem-cell differentiation. Na- 
ture, 2004, 429(6987): 41-46 [DOI]

12 Stainier D. No stem cell is an islet (yet). N Engl J Med, 2006, 354(5): 521-523[DOI]

13 Teta M, Rankin M M, Long S Y, et al. Growth and regeneration of adult beta cells does not involve specialized progenitors. Dev Cell, 2007, 12(5): 817-826[DOI]

14 Shiroi A, Yoshikawa M, Yokota H, et al. Identification of insulin-producing cells derived from embryonic stem cells by zinc-chelating dithizone. Stem Cells, 2002, 20(4): 284-292[DOI]

15 Cornelius J G, Tchernev V, Kao K J, et al. In vitro-generation of islets in long-term cultures of multipotent stem cells from adult mouse pancreas. Horm Metab Res, 1997, 29(6): 271-277

16 Peck A B, Cornelius J G, Schatz D, et al. Generation of islets of langerhans from adult pancreatic stem cells. J Hepatobiliary Pancreat Surg, 2002, 9(6): 704-709 [DOI]

17 Zulewski H, Abraham E J, Gerlach M J. Multipotential nestin-positive stem cells isolated from adult pancreatic islets differentiate ex vivo into pancreatic endocrine, exocrine, and hepatic phenotypes. Diabetes, 2001, 50(3): 521-533 [DOI]

18 Wu F, Jagir M, Powell J S. Long-term correction of hyperglycemia in diabetic mice after implantation of cultured human cells derived from fetal pancreas. Pancreas, 2004, 29(1): E23-29 [DOI]

19 Yatoh S, Dodge R, Akashi T, et al. Differentiation of affinity-purified human pancreatic duct cells to beta-cells. Diabetes, 2007, 56(7): $1802-1809 \underline{\text { [DOI] }}$

20 Marshak D R, Gardner R L, Gottlieb D. Stem Cell Biology. 3rd ed. New York: Cold Spring Harbor Laboratory Press, 2001.499

21 Bonner-Weir S, Weir G C. New sources of pancreatic beta-cells. Nat Biotechnol, 2005, 23(7): 857—861[DOI]

22 Teitelman G, Alpert S, Polak J M, et al. Precursor cells of mouse endocrine pancreas coexpress insulin, glucagon and the neuronal proteins tyrosin hyaroxylase and neuropeptide Y, but not pancneatic polypeptide. Development, 1993, 118(4): 1031-1039

23 Yang K M, Li A D, Mei Y, et al. Islet formation and regeneration. Chin Med Sci J, 2006, 21(1): 27-32

24 Maria-Engler S S, Correa-Giannella M L, Labriola L, et al. Co-localization of nestin and insulin and expression islet cell markers in long-term human pancreas tic nestin-posive cell cultures. J Endocrinol, 2004, 183(3): 455-467[DOI]

25 Peters K, Panienka R, Li J, et al. Expression of stem cell markers and transcription factors during the remodeling of the rat pancreas after duct ligation. Virchows Arch, 2005, 446(1): 56-63[DOI]

26 Taguchi M, Otsuki M. Co-localization of nestin and PDX-1 in small evaginations of the main pancreatic duct in adult rats. J Mol Histol, 2004, 35(8-9): 785-789 $\underline{\text { [DOI] }}$

27 Danner S, Kajahn J, Geismann C, et al. Derivation of oocyte-like cells from a clonal pancreatic stem cell line. Mol Hum Reprod, 2007, 13(1): $11-20 \underline{\text { [DOI] }}$

28 Seaberg R M, Smukler S R, Kieffer T J, et al. Clonal identification of multipotent precursors from mouse pancreas that generate neural and pancreatic lineages. Nat Biotechnol, 2004, 22(9): 1115-1124[DOI]

29 Davani B, Ikonomou L, Raaka B M, et al. Human islet-derived precursor cells are mesenchymal stromal cells that differentiate and mature to hormone-expressing cells in vivo. Stem Cells, 2007, 25(12): 3215-3222[DOI]

30 Kruse C, Kajahn J, Petschnik A E, et al. Adult pancreatic stem/progenitor cells spontaneously differentiate in vitro into multiple cell lineages and form teratoma-like structures. Ann Anat, 2006, 188(6): 503-517 